\title{
Gut microbiota reveals the environmental adaption in gastro-intestinal tract of wild boar in karst region of Southwest China
}

Heqin CaO ${ }^{1,2+}$, Xiongwei Yang ${ }^{1,2+}$, Caichun Peng ${ }^{1,2}$, Yeying Wang ${ }^{3}$, Qunyi Guo ${ }^{4}$ and Haijun $\mathrm{Su}^{1,2^{*}}$

\begin{abstract}
Background: Gut microbes has become one of the research hotspots in animal ecology, playing an important role in monitoring dietary adaptation and health status of host. However, there are few studies on the gut microbiota in the stomach, smallintestine (ileum), and large intestine (cecum, colon, and rectum) of wild boar.

Results: Alpha diversity and Beta diversity showed there were significant differences in the abundance and distribution of microbes in gastrointestinal tract of wild boar. Firmicutes and Bacteroidetes were the most dominant phyla in stomach, cecum, colon and rectum of wild boar, while Proteobacteria and Firmicutes were the most dominant in ileum. At genus level, there were different leading genera in stomach (Prevotella and Lactobacillus), small intestine (Escherichia-Shigella and Lactobacillus), and large intestine (Ruminococcaceae_UCG-005, Christensenellaceae_R-7_group, and Escherichia-Shigella). PICRUSt function predictive analysis suggested that there were significant differences in microbial metabolic pathways among five locations of wild boar.
\end{abstract}

Conclusions: This study comprehensively revealed the differences in composition of microbial community in gastrointestinal trac of wild boar. Future work links microbes with the metabolites to accurately reveal the health of wild boar.

Keywords: Wild boar, $16 \mathrm{~S}$ rRNA gene sequencing, Gut microbiota, Gastrointestinal tract, Environmental adaption, Karst mountainous area

\section{Background}

Wild boar (Sus scrofa), belonging to Suidae, Sus, which is widely distributed in Europe, Asia, and Africa (Genov 2006, Isaacson and Kim 2012), is one of the important resource mammals (Liu et al. 2011). S.s.Chirodentus, main subspecies in south of Yangtze River in China, which is the main large ungulate with increasing number in the karst mountain forest ecosystem of China, and its harm is increasing year by year without good prevention and

\footnotetext{
*Correspondence: hjsu@gzu.edu.cn

${ }^{\dagger} \mathrm{Heqin} \mathrm{Cao}$ and Xiongwei Yang contributed equally to this work.

${ }^{2}$ Research Center for Bio-diversity and Nature Conservation, Guizhou University, Guiyang 550025, China

Full list of author information is available at the end of the article
}

control strategies (Su et al. 2018). Although karst areas in Southwest China suffer from the worst rocky desertification, wild boars can also adapt to the harsh environment and breed quickly. However, there are few researches on exploring the causes of this phenomenon.

Gut microbiota plays a key role in maintaining the healthy growth and development of animals (Fernando et al. 2010; McCoy et al. 2013). Different hosts will form their own gut microbiome in the process of adapting to their growth environment, so as to adapt to the surrounding environment. $16 \mathrm{~S}$ rRNA sequencing has been widely used to study the composition and abundance gut microbiota of the human and animals (Crespo-Piazuelo et al. 2018; David et al. 2014). Researchers have also revealed the stability of the gut microbiota is closely 
related to the health of the population (Guo et al. 2019; Suchodolski et al. 2015). Therefore, the study on the gut microbiota diversity of wild boar in the stomach, small intestine (ileum) and large intestine (cecum, colon, and rectum) can better reveal the function in each location and relations between gut microbiota and population distribution.

However, most of the current studies on the gut microbiota of wild boar are based on fecal microbiota. Due to the different microbial composition in different intestinal regions, fecal microbiota cannot fully reflect the microbial composition of the whole intestine (McCoy et al. 2013; Suen et al. 2011). In this study, 16S rRNA high-throughput sequencing technology was used to analyze and reveal the composition and function of gut microbiota in the stomach, small intestine (ileum) and large intestine (cecum, colon, and rectum) of wild boar in karst area of Southwest China, and to predict the function of the microbes, hoping to understand the role of the microbes in extensively environmental adaptation of wild boar. Our findings provide a reliable basis for formulating scientific and effective prevention and control countermeasures of wild boar, and maintaining the balance of ecological system.

\section{Results}

A total of 2,903,264 valid sequences were obtained from the wild boar gut microbiome by Illumina MiSeq sequencing. Overall, 1664 OTUs were identified from all samples analyzed. Rarefaction curves approached the saturation phase in all samples (Fig. 1).

\section{The microbiota diversity in the gastrointestinal tract of wild boar}

Venn analysis showed that the number of OTU in cecum and colon was the largest (1367 and 1363, respectively), the number of OTU in rectum and ileum was next (874 and 843, respectively), and the number of OTU in stomach was the least (246) (Fig. 2). The unique OTU in stomach, ileum, cecum, colon, and rectum accounted for only $1.74 \%, 5.47 \%, 3.85 \%, 4.15 \%$, and $0.54 \%$ of the total. Besides, the OTU shared by the five locations accounted for $4.81 \%$ of the total, while the OTU shared by cecum and colon was as high as $58.89 \%$.
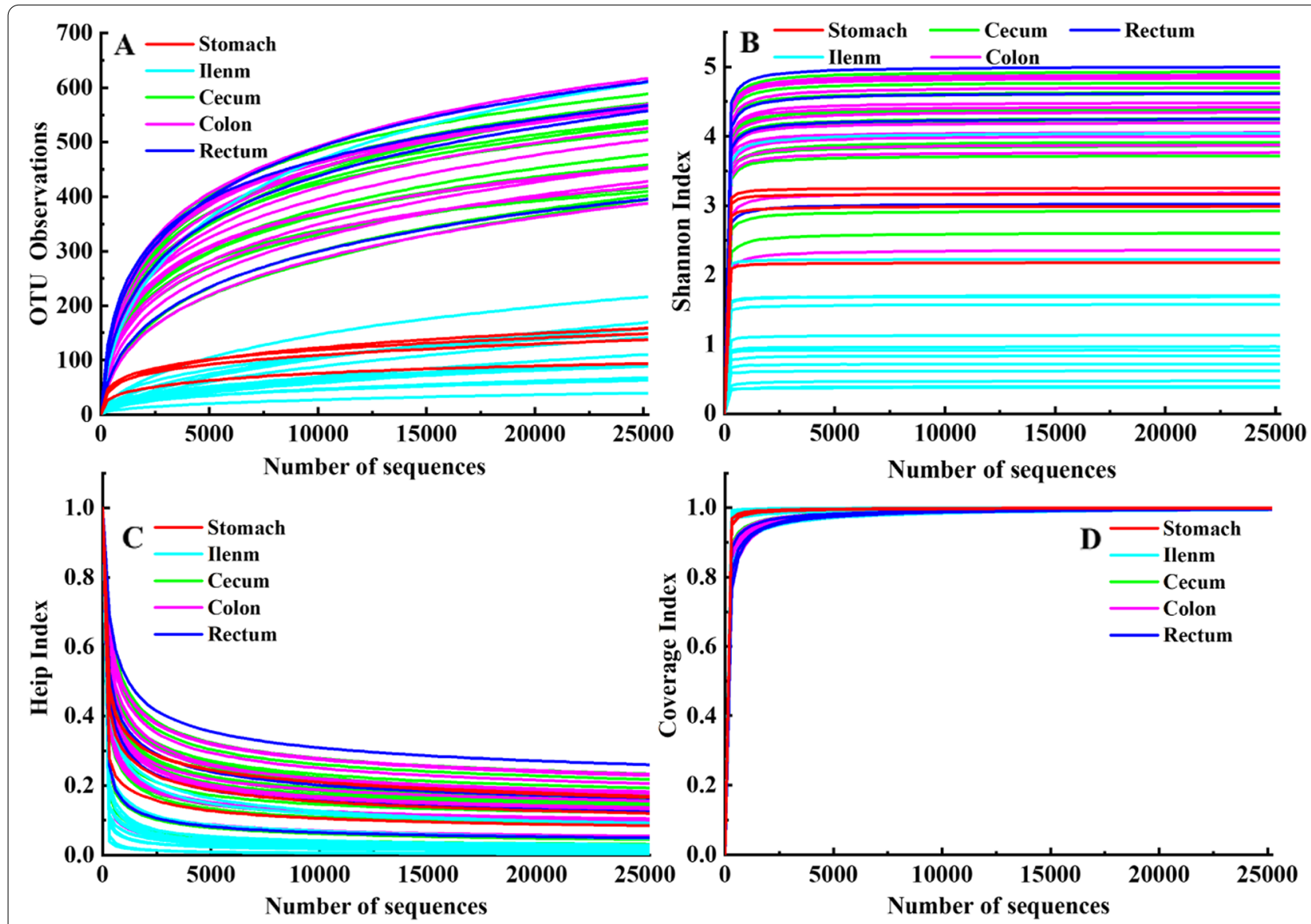

Fig. 1 Bacterial richness curve (A), Shannon curve (B), Heip index curve (C), and coverage index curve (D) of gastrointestinal tract of wild boar 


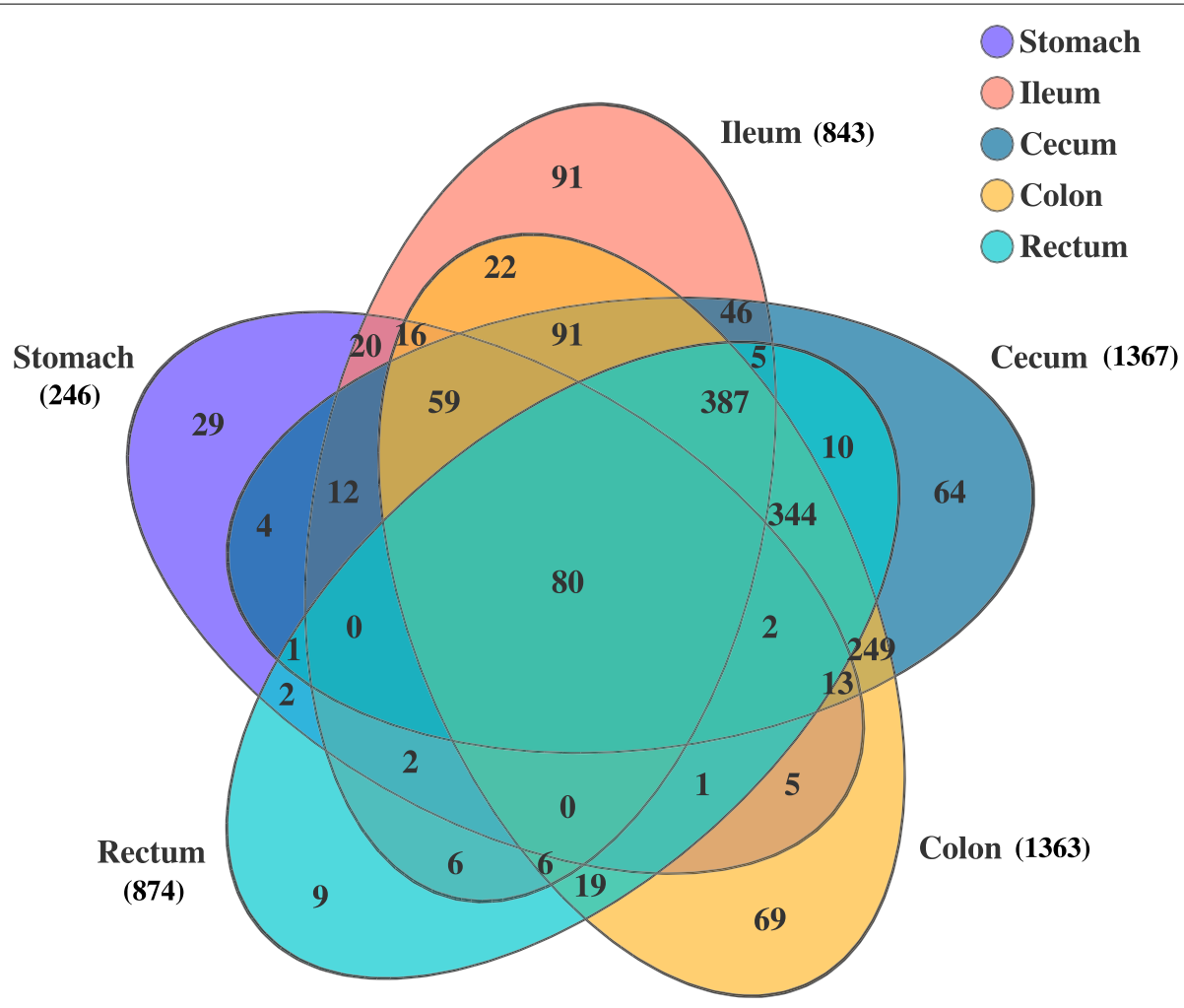

Fig. 2 Venn diagrams for microbiota OTUs compositions in gastrointestinal tract of wild boar
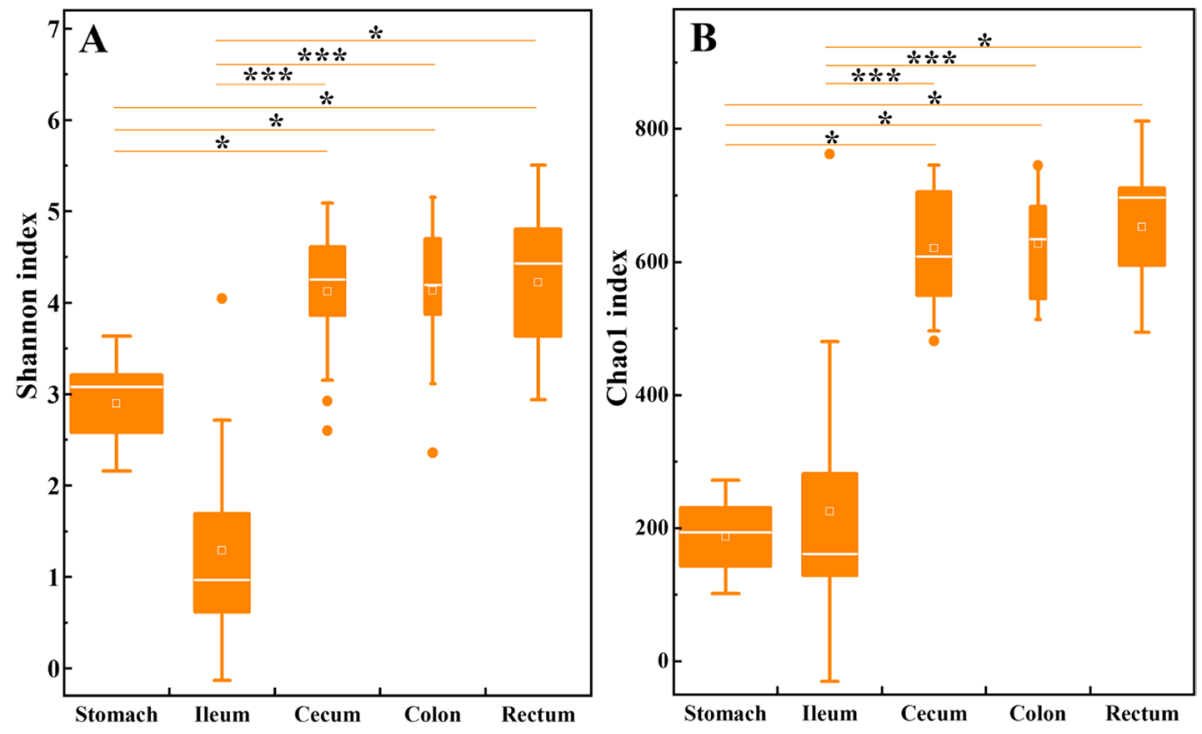

Fig. 3 The microbiota diversity estimated by Chao1 (A), Shannon index (B) in the gastrointestinal tract of wild boar. Note: The horizontal line in the box represents the median, the little white square represents the mean, the error line represents the SD value, $0.01<p<0.05$ was marked as ${ }^{*} p<$ 0.01 was marked as *** 
Alpha diversity showed that there were significant differences in the distribution of microbes in gastrointestinal tract of wild boar (Fig. 3). The microbes in cecum, colon and rectum were similar and whose microbiota diversity was higher than that in stomach and ileum, and ileum had the lowest microbiota diversity. There were no significant differences in Chaol index among stomach with ileum, cecum with rectum, and colon with rectum $(p>0.05)$. Nevertheless, the Chaol index of stomach with cecum and colon with rectum were all significantly different $(p<0.05)$. Chiefly, the Chao1 index of the ileum is extremely different from that of the cecum and colon $(p<0.05)$. In addition, there were no significantly different in Shannon index between cecum with colon, and cecum with rectum $(p>0.05)$. However, Shannon index of the stomach was significantly different from that of the cecum, colon, and rectum $(p<0.05)$, and Shannon index of the ileum is extremely different from that of the cecum, colon, and rectum $(p<0.05)$.

PCoA analysis results showed that the samples of each location were aggregated together. There were significantly clustered in cecum, colon and rectum samples. However, the stomach and ileum samples were obviously scattered in different areas (Fig. 4).

\section{The microbiota composition in the gastrointestinal tract of wild boar}

A total of 23 bacteria phyla were identified from 53 gastrointestinal tract samples of wild boar (Fig. 5). Firmicutes, Bacteroidetes, Proteobacteria, Actinobacteria, Verrucomicrobia, Fusobacteria, and Spirochaetes were the main bacterial components of wild boar. Firmicutes was the most dominant phylum in the stomach (40.83\%), cecum $(61.55 \%)$, colon $(62.24 \%)$, and rectum (61.05\%). However, the largest predominant phylum in the ileum was the Proteobacteria (53.49\%). Bacteroidetes was the second most abundant bacteria phylum followed by Firmicutes, accounting for $23.53 \%$ (stomach), $19.23 \%$ (cecum), $18.37 \%$ (colon), and $18.69 \%$ (rectum), respectively. However, the second abundant phylum in ileum was Firmicutes (33.58\%). The dominant bacteria components in stomach, accounting for $98.33 \%$, were

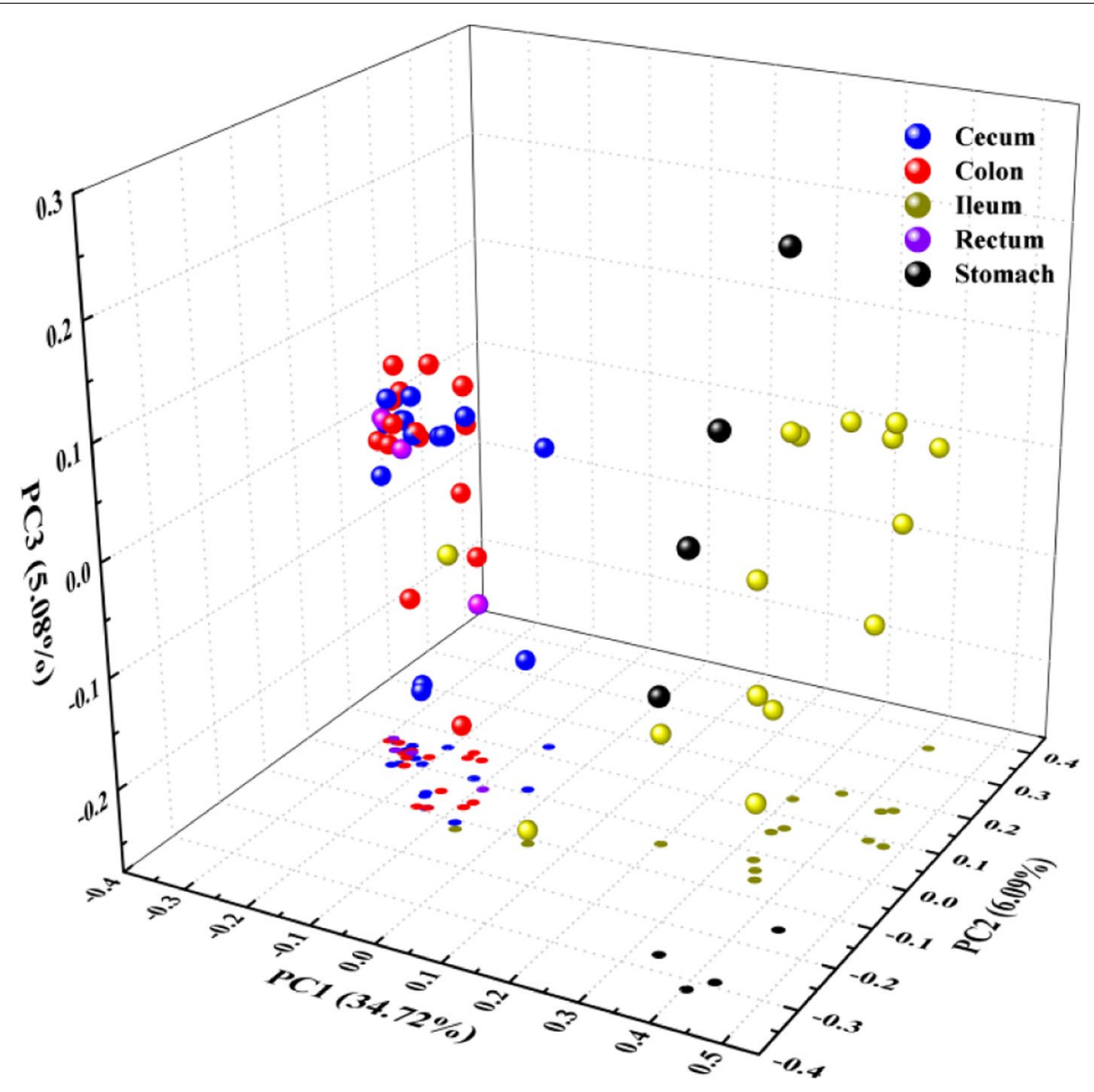

Fig. 4 PCoA analysis of wild boar 


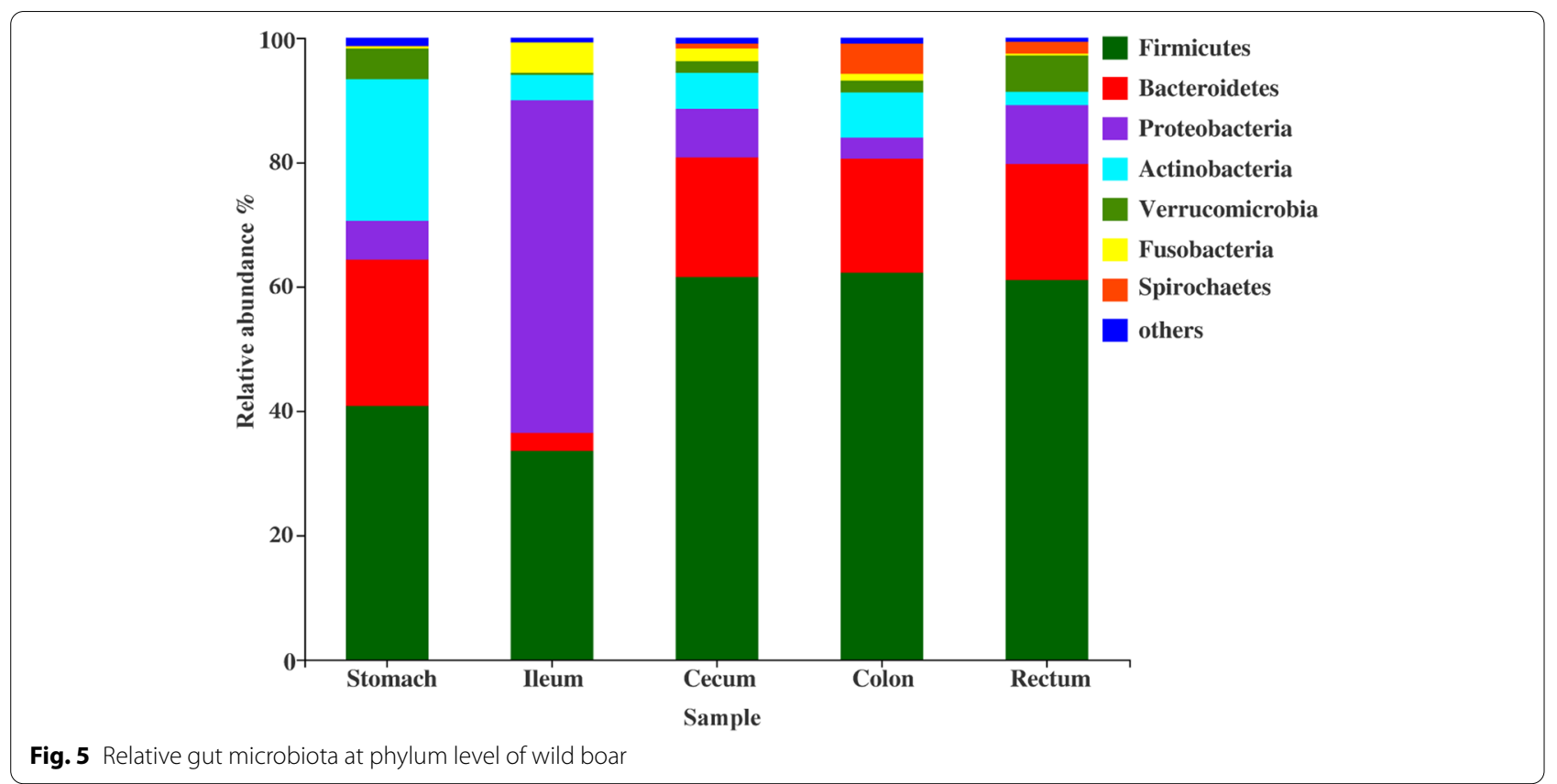

Firmicutes, Bacteroidetes, Actinobacteria, Proteobacteria, and Verrucomicrobia; in ileum (96.04\%) were Proteobacteria, Firmicutes, Fusobacteria, and actinobacteria; in cecum (96.39\%) were Firmicutes, Bacteroidetes, Proteobacteria, Actinobacteria, and Fusobacteria; in colon (96.1\%) were Firmicutes, Bacteroidetes, Actinobacteria, Spirochetes, and Proteobacteria; in rectum (95.06) were Firmicutes, Bacteroidetes, Proteobacteria, and Verrucomicrobia. The microbial composition in colon was significantly different for Firmicutes and Spirochaetes, and the relative abundance was significantly higher than other four locations $(p<0.05)$. And the relative abundance of Proteobacteria in ileum and Bacteroidetes in stomach was significantly different compared with other locations $(p<0.05)$.

At genus level, there were 15 dominant genera components of wild boar (Fig. 6), such as Escherichia-Shigella, Lactobacillus, Terrisporobacter, Bifidobacterium, Fusobacterium, and Pseudoscardovi. The three dominant genera with the highest relative abundance in the stomach were Prevotella (21.40\%), Lactobacillus (12.89\%), and Pseudoscardovia (8.30\%). The predominant genera in ileum were Escherichia-Shigella (50.93\%), Lactobacillus (15.63\%), and Terrisporobacter (7.51\%). The relative abundance in cecum, colon, and rectum was similar, and the three most abundant genera were Ruminococcaceae_UCG-005, Christensenellaceae_R-7_group, and Escherichia-Shigella. There were some differences in stomach, ileum, cecum, colon and rectum. Prevotella was the representative bacterium of stomach, and its relative abundance was far greater than of other locations. Besides, Treponema_2 was just found in the cecum, colon, and rectum, and it was most abundant in the colon. Furthermore,19 genera showed significant differences among five locations of wild boar, including Christensenellaceae_R-7_group, Ruminococcaceae_UCG005, Ter-risporobacter, Prevotella_7, Pseudoscardovia, Bacteroides, and Alloprevotella.

\section{LEfSe analysis of wild boar}

To further explore the differences of gut microbiota communities among five locations, the LEfSe (linear discriminant analysis effect size) analysis was performed at genus level. The results revealed that a total of 44 biomarkers with statistical differences were detected by LEfSe (16 in stomach, 9 in ileum, 4 in cecum, 7 in colon, 8 in rectum). The most of significant biomarkers in five locations were distributed in Firmicutes, taking up $60.87 \%$ (Fig. 7). The abundance of bacteria in stomach was significantly higher than that of other locations, including Prevotella, Pseudoscardovia, Olsenella, Dialister, Mitsuokella, Megasphaera, and Acetitomaculum. The abundance of bacteria in ileum was significantly different from that in other locations, including Lactobacillus, Terrisporobacter and Escherichia-Shigella and Bacteroides. Ruminococcaceae_UCG-005, Eubacterium_coprostanoligenes_group, and Alloprevotella enriched in the cecum. In addition, there were three genera with significant differences in the colon, among which unclassified_f Lachnospiraceae, Prevotellaceae_NK3B31_group, and 


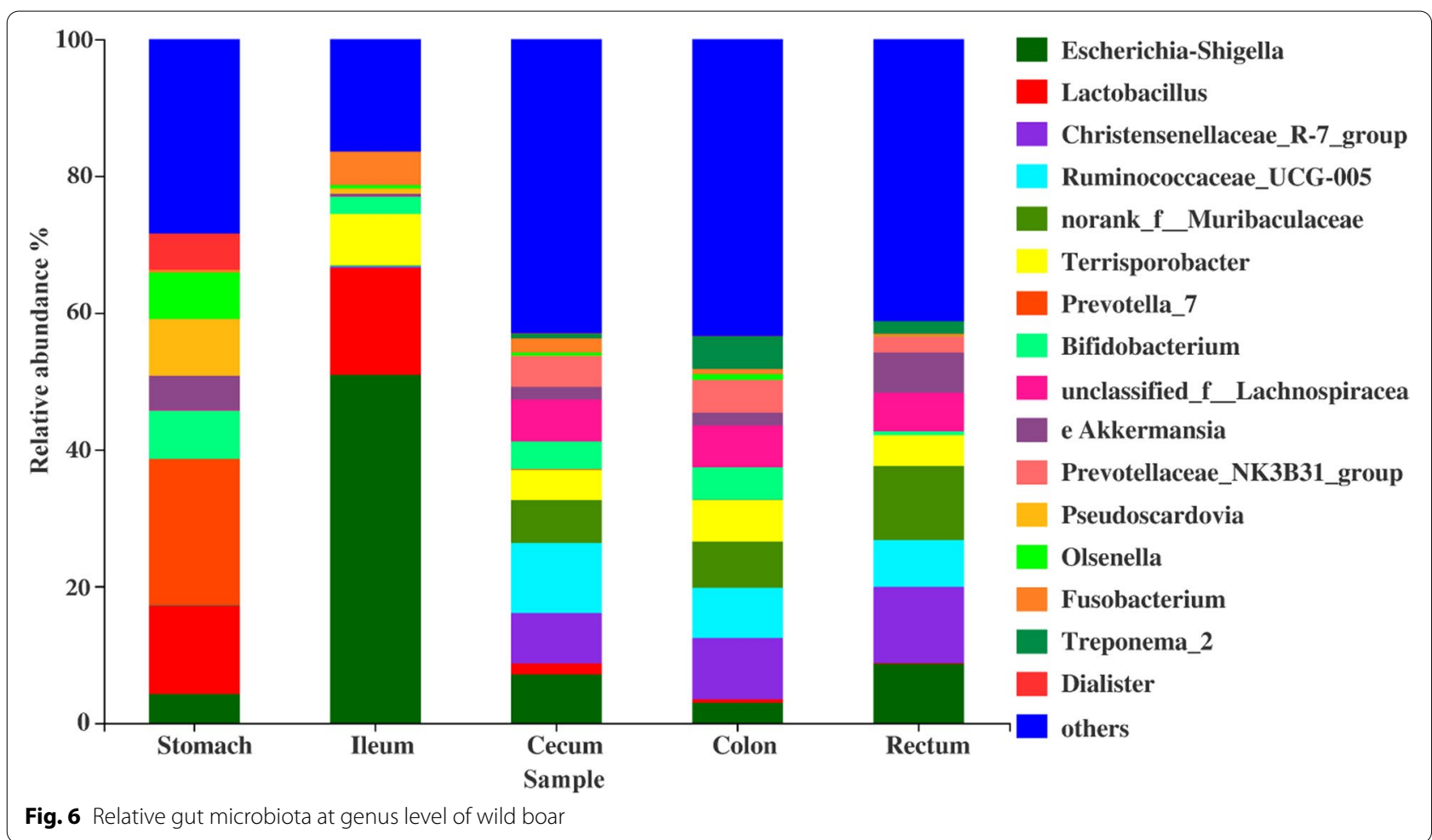

Treponema_2 significantly enriched. We also found that Christensenellaceae_R-7_group, Clostridium_sensu_ stricto_1, Ruminococcaceae_UCG_014, and Turicibacter significantly enriched in the rectum.

\section{PICRUSt function prediction}

By analyzing the differences of KEGG metabolic pathways, we can detect differences in metabolic pathways of function microbial genes between different groups of samples. A total of 46 KEGG metabolic pathways were analyzed. The results showed that there were significant differences in microbial metabolic pathways among five locations of wild boars. Global and overview maps, carbohydrate metabolism, amino acid metabolism, energy metabolism, metabolism of cofactors, and vitamin metabolism were the five highest metabolic pathways (Fig. 8).

This study suggested that there were differences on the function in gastrointestinal tract of the wild boar, and the function of bacterial community in cecum, colon, and rectum is more similar than that in stomach and ileum. As shown in Fig. 9, genes related to starch and sucrose metabolism, fructose and mannose metabolism, tyrosine metabolism, vitamin B6 metabolism, lipoarabinomannan (LAM) biosynthesis, and carotenoid biosynthesis are significantly enriched in the stomach. Ileum microorganisms have rich functions in lipopolysaccharide biosynthesis, degradation of aromatic compounds, glutathione metabolism, fatty acid degradation, steroid hormone biosynthesis, and unsaturated fatty acid biosynthesis. The microbiota in the large intestine (cecum, colon, and rectum) of wild boar function more closely, involved in fatty acid metabolism, fatty acid biosynthesis, biotin metabolism, synthesis and degradation of ketone bodies, glycosaminoglycan degradation, porphyrin, and chlorophyll metabolism, histidine metabolism, valine leucine, and isoleucine biosynthesis function associated with microbial abundance.

\section{Discussion}

There have been several studies about the gut microbial composition of wild boar based on 16S rRNA gene sequencing (Huang et al. 2020; Wang et al. 2020). However, there are few reports about wild boar 16S rRNA gene sequencing analysis that can reveal the microbial community structure in different gut locations. In this study, we compared microbial community structure in five gastrointestinal locations, characterized the microbiome from anterior to posterior, and investigated the potential relationship of gut microbiome with function by high-throughput sequencing. To our knowledge, this is the first report about the comprehensive analysis of microbial community structure in stomach, small intestine (ileum), and large intestine (cecum, colon and 


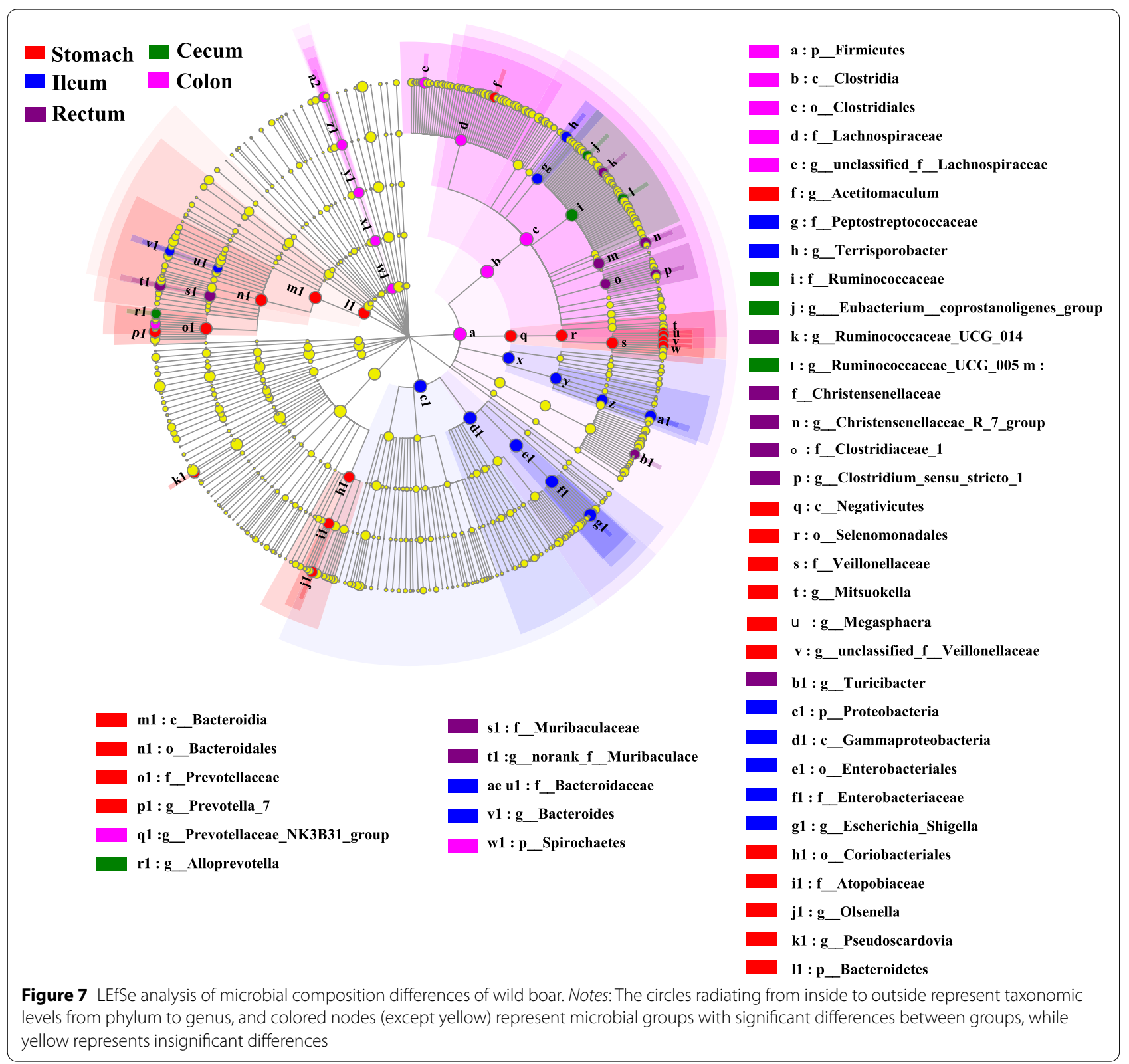

rectum) of wild boar and predicts their function with microbes.

\section{The microbiota diversity of wild boar and comparison with other ungulates}

Gut microbiota plays a key role in maintaining the healthy growth and development of animals. And there is a complex dynamic balance between the host and gut microbes. A stable gut ecological environment contributes to the synthesis, digestion and absorption of nutrients by animals. It is also of great significance in metabolism, immune regulation and a variety of gut diseases (Chi et al. 2014; Huang et al. 2018; Xiong et al. 2015; Zhu et al. 2016). In our study, a total of 394 genera, 160 families, 23 phyla were identified, and the abundance and diversity of microbiota were higher than those of the captive wild boars and domestic pigs from Xinyang County, Henan Province (163 genera, 17 phyla) and Chongqing City (118 genera, 13 phyla) (Wang et al. 2020; Yang et al. 2020; Yang et al. 2016). This may be due to the difference in gut microbiota caused by different breeds (Huang et al. 2020), or the difference between captive and wild environments. In this study, the food sources of wild boar in karst region of Southwest China are more complex and diverse, almost 


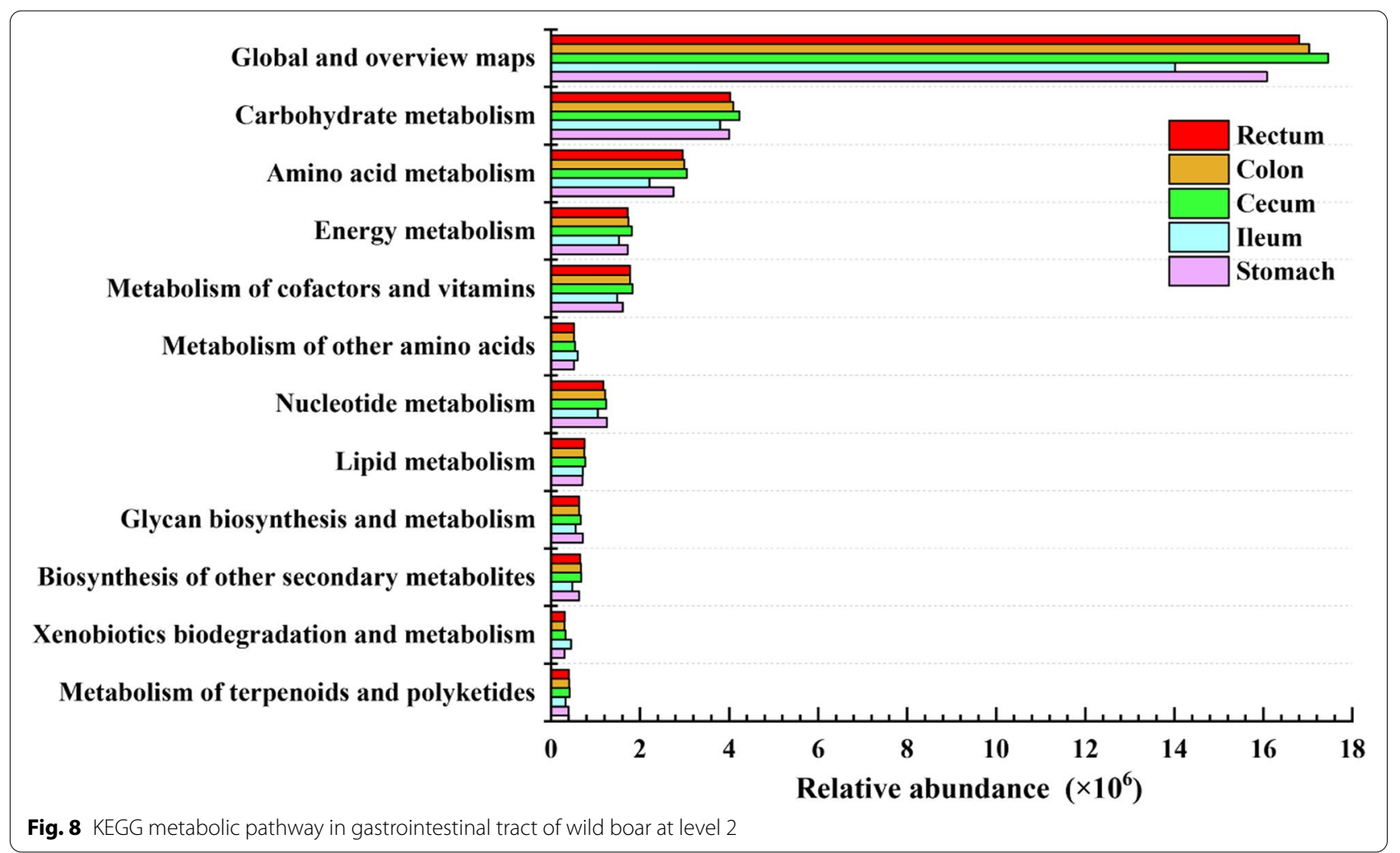

including higher plants (roots, stems, leaves, seeds and fruits), crops (corn, soybeans), invertebrates (snails, earthworms, insects, spiders, millipedes, crabs, and centipede) and vertebrates (rodents, artiodactyla, birds, amphibians, and fish), and some algae and fungi, and even including inorganic materials such as plastic and stone. Previous studies have also shown that the complexity of diet sources may lead to a high degree of microbiota diversity of animal. Omnivorous cattle consume a greater variety of food sources than herbivorous cattle, resulting in an increase in the microbiota diversity with the increase in dietary (Lau et al. 2018).

The composition of bacterial community structure in the gastrointestinal system of wild boar varies with its location. The diversity analysis showed that the diversity of bacterial community in the stomach, small intestine (ileum) and large intestine (cecum, colon and rectum) was significantly different. The microbiota diversity in small intestine was lower than that in stomach, and the Chaol and Shannon indexes in stomach and small intestine were significantly lower than that in large intestine $(p<0.05)$, indicating that the bacterial community structure in large intestine of wild boar is more complex. Some studies have proved that the number of gut microbes of various fishes increases gradually from front to back (Ye et al. 2014). The stomach is an important digestive organ and digests most of the food by mixing it with gastric juices thoroughly through peristalsis. The small intestine is mainly responsible for enzyme digestion and absorption of starch, and the large intestine provides nutrients for the body through bacterial fermentation of non-starch polysaccharides (McCoy et al. 2013; Suen et al. 2011), thus gathering a higher richness and diversity of gut microbes. High microbial diversity is a benign sign of animal intestinal health. The intestinal ecosystem of wild boar with high bacterial abundance not only helps the body resist external influences, but also plays a key role in preventing pathogen colonization and maintaining intestinal homeostasis (Huang et al. 2020).

\section{Gut microbiota of wild boar and comparison with other ungulates}

This study suggested that compositions of microbiome throughout the gastrointestinal system vary with location. Isaacson(Isaacson and Kim 2012) and Yang(Yang et al. 2016) reported that Firmicutes represents in the ileum more than $95 \%$ and $65 \%$, respectively, and Firmicutes and Bacteroidetes occupy greater than $90 \%$ in the cecum of pigs. However, in this study, Proteobacteria was the highest bacterial phylum in the ileum, and Firmicutes was the second highest phylum that accounted for $33.58 \%$ of relative abundance. Firmicutes and Bacteroidetes were two 


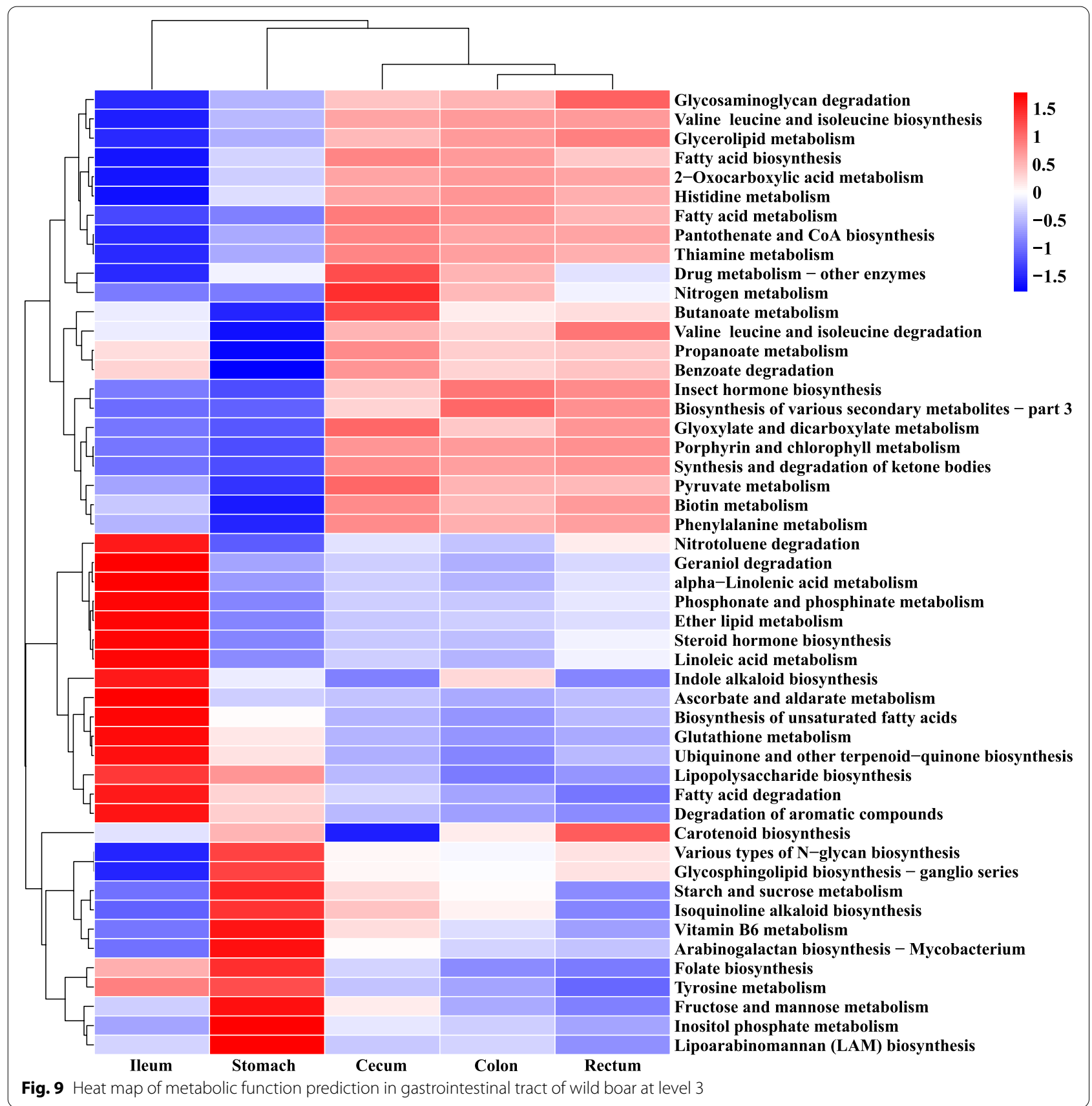

dominant phyla in the cecum, which showed $61.55 \%$ and 19.23\%. The reason for this difference may be that the diet and breed of wild boars and domestic pigs differ greatly, leading to this result. Firmicutes, Bacteroidetes, Proteobacteria, Actinobacteria, Verrucomicrobia, Fusobacteria, and Spirochetes were the major phyla in gastrointestinal system of wild boar. This is similar to the gut microbiota distribution of most mammals such as domestic pigs, captive wild boars, Hainan special wild boar, snubnosed monkey (Rhinopithecus bieti), and moose (Alces alces) (Ishaq and Wright 2014; Wang et al. 2020; Wu et al. 2010; Yang et al. 2020; Yang et al. 2016). Firmicutes and Bacteroidetes are widely present in herbivorous animals, especially in ungulates. For example, the proportion of Firmicutes and Bacteroidetes of sika deer (Cervus Nippon) and moose is more than $90 \%$ (Guan et al. 2017; Ishaq and Wright 2014). It has been found that the core microbiota of Sichuan snub-nosed monkey (Rhinopithecus Roxellana) in Primates (Wang et al. 2015), giant panda (Ailuropoda melanoleuca) in Carnivora (Zhu et al. 2016; 
Zhu et al. 2011), and mice in Rodentia (Weldon et al. 2015) also were Firmicutes and Bacteroidetes, which may be due to the fact that these herbivores all live on crude fiber requiring digestion and absorption a large amount of cellulose, hemicellulose, and other nutrients that are difficult to degrade. In this study, the relative abundance of Firmicutes and Spirochaetes in colon, Proteobacteria in ileum and Bacteroidetes in stomach was significantly different compared with other four locations $(p<0.05)$, but other phyla showed no significant difference. It has been reported that the bacteria of the phylum Spirochaetes occupy a certain proportion of microbes in captive sika deer (Guan et al. 2017), similar to the results of this study, wild boar accounted for $4.85 \%$.

As the most major phylum in colon of wild boar, the relative abundance of Firmicutes (62.24\%) was significantly different than other locations $(p<0.05)$. Numeric studies have reported that Firmicutes is the most predominant phylum of animals, such as sika deer (Guan et al. 2017), pigs (Crespo-Piazuelo et al. 2018), and moose (Ishaq and Wright 2014). Firmicutes can not only decompose cellulose into volatile fatty acids available to the host, improving the nutrient utilization of the host, but also regulate $\mathrm{T}$ cells to improve host immunity, prevent intestinal inflammation, and maintain intestinal microbial ecological balance (Fernando et al. 2010; Guan et al. 2017). However, Wu et al found Firmicutes might not always be the most dominant phylum that could be ranked after Bacteroidetes in dhole (Cuon alpinus) (Wu et al. 2016). We observed that Firmicutes was not the most major phylum in ileum of wild boar, but Proteobacteria. This may be related to the fact that each gut location is functionally diverse.

Bacteroidetes is another dominant phylum among mammalian animals, which was significantly higher $(p$ $<0.05)$ in stomach of wild boar $(23.53 \%)$ compared with other locations. As a crucial phylum in gut microbiota, Bacteroidetes was functional for degradations of high molecular weight substances and carbohydrates that from intestinal secretions, which can improve the utilization rate of carbohydrate, protein, and other substances and enhance host immunity (Becker et al. 2014; Ma et al. 2014; Salyers et al. 1977; Thoetkiattikul et al. 2013). The Firmicutes/Bacteroidetes ratio was different in different location of wild boar, but lowest in ileum. Mariat (Mariat et al. 2009) found the Firmicutes/Bacteroidetes ratio evolved during different stages of life in human gut microbiota that represented distinct diversity of bacterial communities and digestive ability.

Notably, the relative abundance of Proteobacteria in ileum of wild boar in this study was higher than that of Firmicutes, and the relative abundance of Proteobacteria $(p<0.05)$ was significantly higher in ileum of wild boar.
Previous studies showed that Proteobacteria is the main microbes of giant panda (Ailuropoda melanoleuca) (Xue et al. 2015), red panda (Ailurus fulgens) (Zeng et al. 2018), North China leopard (Panthera pardus japonensis) (Hua et al. 2020), and Amur tiger (Panthera tigris) (He et al. 2018). It has been found that Proteobacteria contain a variety of pathogenic bacteria, which is a sign of unstable gut microbiota (Shin et al. 2015). It can assist in degrading lignin, and the high abundance of Proteobacteria may be in order to better cope with the complex feeding habits of wild boar (Fang et al. 2012), so whether the high abundance of Proteobacteria is beneficial or harmful to wild boar needs to be further verified.

In addition, we also noticed Spirochaetes, which occupied $4.07 \%$ of microbiota composition in the colon and had a significantly higher relative abundance than that in other locations of wild boar. Yang (Yang et al. 2016) found Spirochaetes, taken up 2.7\% of abundance in the cecum, had a significantly higher relative abundance than that in the small intestine. Abdel-Moein (Abdel-Moein et al. 2015) revealed that Spirochaetes, which is generally considered a potential pathogen, may cause a variety of chronic infectious diseases.

At genus level, as the previous research of pigs, Looft (Looft et al. 2014) found that differences of bacterial compositions between the ileum and colon were the results of the dominant genera Anaerobacter and Turicibacter in the ileum, and Prevotella, Oscillibacter, and Succinivibrio in the colon. And Yang (Yang et al. 2016) reported that Clostridium was the top genus in the ileum and showed significantly different abundance between the ileum and cecum, while Prevotella was most abundant and significantly enriched in the cecum. However, in this study, the top genus in ileum, cecum and colon was Escherichia-Shigella, Ruminococcaceae_UCG-005, and Christensenellaceae_R-7_group, respectively, and showed significantly different abundance, similar with Wang's results on the proportions of Christensenellaceae_R-7_ group and Rikenellaceae_RC9_gut_group in the Hainan special wild boar. This discrepancy may be due to different breed, diet, environment factors and the genetic characteristics of crude fiber intake in wild boar.

The results showed that Prevotella, Lactobacillus, and Megasphaera were dominant in the stomach. Lactobacillus as probiotics exist widely in the intestines of animals, not only can break down carbohydrates and inhibit the growth of pathogen, also can activate the immune cells to improve immunity (Petrova et al. 2015; Ruggiero 2014). Prevoella plays an important role in decomposing cellulose, promoting protein decomposition and improving the degradation capacity of cellulose (Jin et al. 2019). Megasphaera converts lactic acid to propionic acid, reducing the risk of mammalian acidosis (Henning et al. 
2010). This is extremely important for wild boars, which are often driven to high levels of exercise by hunting or avoiding predators in the wild.

In the ileum, the abundance of Escherichia-Shigella, Lactobacillus and Bacteroides were dominant bacteria, Consistent with Wang and Liu's research results on cattle and sheep, respectively. Yuan (Yuan et al. 2019) and Fang' research (Fang et al. 2012) results on gut microbiota of sun bear (Helarctos Malayanus) and giant panda, respectively. Escherichia-Shigella is a pathogen that causes diarrhea in animals. An anaerobic environment conducive to the colonization of obligate anaerobic bacteria will be formed in the intestinal tract, which will lead to increased abundance of Lactobacillus and Bifidobacterium in the intestinal tract (Li et al. 2012). In this study, the high proportion of Escherichia-Shigella and Lactobacillus in the ileum, which may be that they are regulating and maintaining the intestinal environment of wild boar (Ruggiero, 2014). Bacteroidetes can facilitate digestion in wild boars (Becker et al. 2014; Ma et al. 2014). In addition, Akkermansia is also the dominant bacteria in ileum, which is a very important probiotic bacteria in animal intestines, and its products can provide energy for ruminants (Suen et al. 2011).

Ruminococcaceae_UCG-005, Escherichia-Shigella, and Christensenellaceae_R-7_group were the dominant bacteria in cecum, colon, and rectum, and some studies have shown that these bacteria are the dominant bacteria in rumen of ruminants (Jin et al. 2019). Ruminococca-ceae_UCG-005 are very important probiotics in animal intestines, which can secrete a large amount of cellulase and hemicellulase to degrade starch and cellulose, and the products can provide energy for the animal body (Suen et al. 2011). Studies in pigs found that Christensenellaceae_R-7_group help digest soluble dietary fiber (Tao et al. 2019) and promote intestinal development and barrier function, thus improving the growth performance of pig (Zhang et al. 2020). Christensenellaceae_R-7_ group, with the highest abundance in the rectum, can reduce the probability of obesity in mammals, which is consistent with the characteristics of wild boar with higher lean meat rate and lower fat content. In addition, it is reported that Christensenellaceae_R-7_group and Ruminococcaceae_UCG-005 are related to the production of short-chain fatty acids (SCFA) (Li et al. 2021; Zhang et al. 2020).

In addition, Bacillus in the rectum can transform and degrade lactic acid, so that animals with high levels of exercise can always maintain adequate physical energy for foraging, running, and other activities (Huang et al. 2020). Turicibacter is also positively correlated with dietary cellulose richness, which helps wild boar to adapt to a plant-based diet (Lau et al. 2018). Although the abundance of Bifidobacterium in this study is relatively low, Bifidobacterium plays an important role in inhibiting the proliferation of pathogenic bacteria and pre-venting intestinal inflammation. The high level of Bifidobacterium contributes to the absorption of nutrients and the improvement of disease resistance of wild boar (Di Gioia et al. 2014). Therefore, the rich microbiota in each location of the wild boar has sufficient ability to help its adapted to the harsh natural conditions in the wild.

\section{Gut microbiota and function prediction}

KEGG pathway was used to predict the intestinal microbiome function of wild boar. The results showed that genes related to starch and sucrose metabolism, fructose and mannose metabolism, tyrosine metabolism, vitamin metabolism, folic acid biosynthesis, and carotenoid biosynthesis were significantly enriched in the stomach of wild boar. This is related to the enrichment of Prevotella and Lactobacillus in the stomach, and these two bacteria can provide energy to the animal body during decomposition of the acetic acid, propionic acid, and butyric acid produced by decomposition of the semicellulose, pectin, starch, etc. (Jin et al. 2019; Petrova et al. 2015). The function of the ileum is more closely related to the relationship between fatty polysaccharide biosynthesis, fatty acid degradation, metabolism, phosphoric acid and phosphoric acid metabolism, and unsaturated fatty acids. It may be due to a large number of Lactacillus, Bacillus, and Fusobacterium, which can decompose protein, metabolize carbohydrates (McCoy et al. 2013; Niu et al. 2015). The function of microbial flora in cecum, colon and rectum is relatively close, which associated microbial abundance of fatty acid metabolism, amino acid biosynthesis, carbohydrate metabolism, energy metabolism, etc. This is related to the high abundance of Ruminococcaceae_UCG-005, Prevotella_7, and Christensenellaceae_R-7_group, which requires anaerobic conditions and carbohydrate energy sources in dietary fibers (such as cellulose or xylan). Similar functions have been found in the captive wild boar (Yang et al. 2020). This study suggests that microbes in the small intestine are more inclined to quickly digest, absorb simple carbohydrates such as glucose and maltose. Carbohydrates that are more difficult to decompose enter the cecum and colon, and the bacterial communities such as Akkermansia and Clostridium_sensu_stricto_1 help to decompose and utilize (Suen et al. 2011).

The function prediction corresponds to the abundance of microbiota. Different locations show unique functional and spatial characteristics, which contribute to the rapid degradation of various nutrients, utilization and maintenance of intestinal dynamic balance, which may also be 


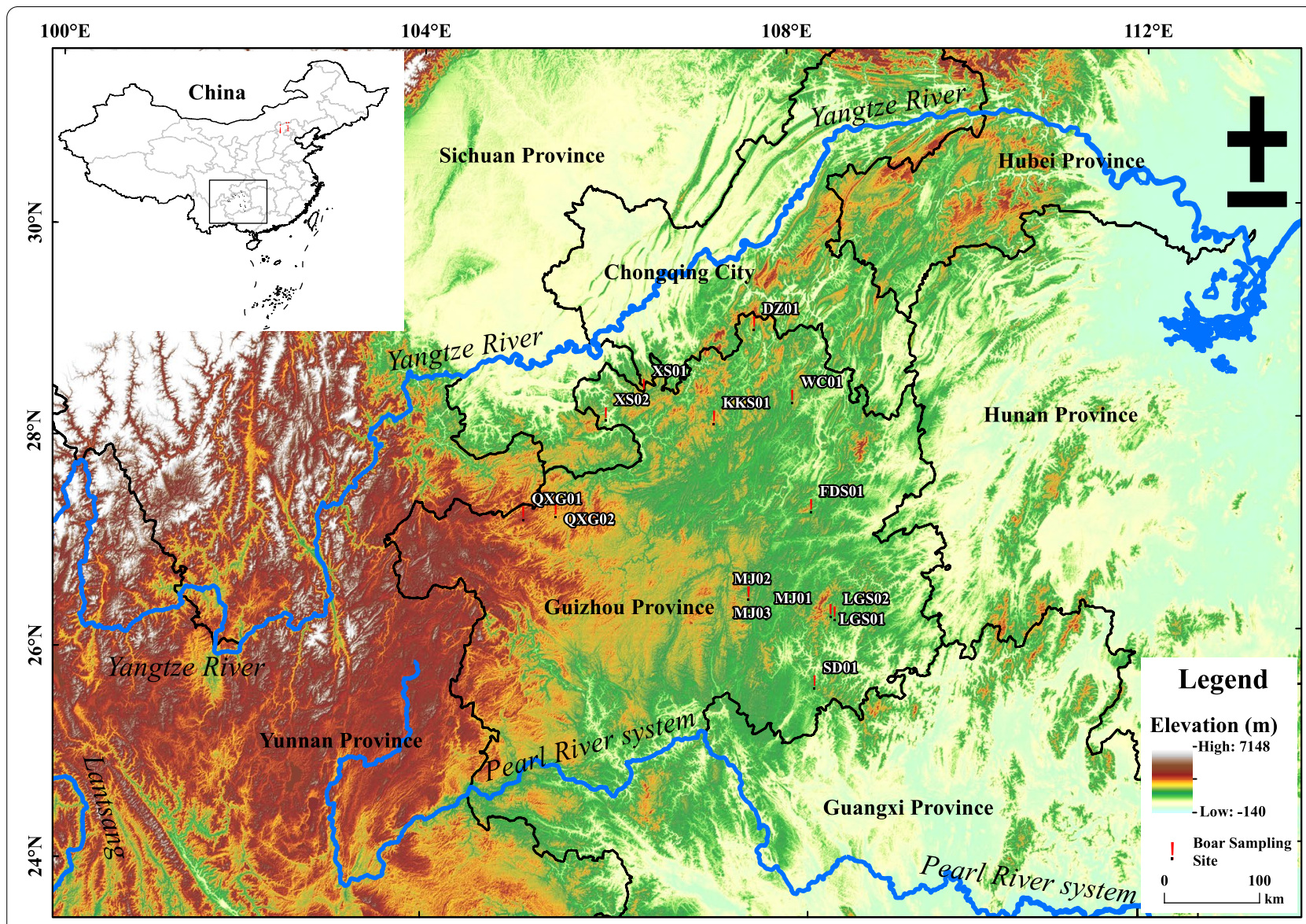

Fig. 10 Sampling location distribution of 15 healthy wild boars

one of the reasons for the wide diet and strong adaptability of wild boar.

\section{Conclusion}

Our study suggested that the composition of bacterial community structure of wild boar varies with gastrointestinal location. To our knowledge, this study is the first to analyze and compare the gut microbiota of wild boar in stomach, small intestine (ileum), and large intestine (cecum, colon, and rectum) worldwide. The results of this study are helpful to develop better prevention and control strategies for wild boar. With the continuous deepening of gut microbiota related research, it is expected to become an effective means to reveal the health of wild boar.

\section{Methods}

\section{Fecal sample collection}

Fifteen healthy wild boars were collected in the forest environment of a karst region in Southwest China, in the winter of 2019 to 2020 . The distribution of the sampled wild boars is shown in Fig. 10, and the detailed sample information is shown in Table S1. After the wild boar was killed, 3-5 g contents of stomach, ileum, colon, cecum, and rectum were collected under sterile conditions and stored at $-80{ }^{\circ} \mathrm{C}$ for further analysis. The hunting and autopsy samples were approved by Guizhou Forestry Bureau and Animal Experiment Ethics Committee of Guizhou University.

\section{$16 \mathrm{~S}$ rRNA microbial community analysis Total DNA extraction and $P C R$ amplification}

Total DNA was extracted from the microbial community according to the instructions of E.Z.N.A. ${ }^{\circledR}$ soil DNA kit (Omega Bio-Tek, Norcross, GA, USA), and the quality of DNA was detected by $1 \%$ agarose gel electrophoresis (voltage $5 \mathrm{~V} / \mathrm{cm}, 20 \mathrm{~min}$ ). DNA concentration and purity were determined by NanoDrop2000. Using extracted DNA as template, 338F (5'-ACTCCTACGGGAGGC AGCAG- $3^{\prime}$ ) and 806R (5'-GGACTACHVGGGTWT CTAAT- $3^{\prime}$ ) were selected as primers for PCR amplification of V3-V4 variable region of 16SrRNA gene. The PCR reaction parameters were as follows: pre-denaturation at 
$95{ }^{\circ} \mathrm{C}$ for $3 \mathrm{~min}, 27$ cycles (denaturation at $95{ }^{\circ} \mathrm{C}$ for 30 $\mathrm{s}$, annealing at $55^{\circ} \mathrm{C}$ for $30 \mathrm{~s}$, extension at $72{ }^{\circ} \mathrm{C}$ for $45 \mathrm{~s}$ ), then stable extension at $72{ }^{\circ} \mathrm{C}$ for $10 \mathrm{~min}$, and finally preservation at $10{ }^{\circ} \mathrm{C}$ (PCR: ABI GeneAmp $\left.{ }^{\circledR} 9700\right)$. PCR reaction system is as follows: $5 \times$ FastPfu buffer $4 \mu \mathrm{L}, 2.5 \mathrm{mM}$ dNTPs $2 \mu \mathrm{L}$, upstream primer $(5 \mu \mathrm{M}) 0.8 \mu \mathrm{L}$, downstream primer $(5 \mu \mathrm{M}) 0.8 \mu \mathrm{L}$, TransStart FastPfu DNA polymerase $0.4 \mu \mathrm{L}$, BSA $0.2 \mu \mathrm{L}$, template DNA $10 \mathrm{ng}$, DdH2O to $20 \mu \mathrm{L}, 3$ replicates per sample. PCR products from the same sample were mixed and purified by $2 \%$ agarose gel electrophoresis using the AxyPrep DNA Gel recovery kit (AXYGEN, USA). The recovered products were detected and quantified using the QuantiFluor ${ }^{\mathrm{TM}}$-ST blue fluorescent quantification system (Promega, USA).

\section{Illumina Miseq sequencing}

NEXTFLEX Rapid DNA-SEQ Kit was used for library construction: (1) "Y" joint link; (2) use magnetic beads to remove self-connecting segments; (3) enrichment of library templates by PCR amplification; Sodium hydroxide denatures to produce single-stranded DNA fragments; (4) PCR products were recovered by magnetic beads to obtain the final library. Sequencing was performed on Illumina's Miseq PE300 platform (Shanghai Majorbio Bio-Pharm Technology Co., Ltd.).

\section{Statistical analysis}

Statistical comparisons and graphic were analyzed by the Statistical Package for Social Science program (SPSS22.0, Chicago, USA) and R 4.11. $p<0.05$ was considered statistically significant.

\begin{abstract}
Abbreviations
rRNA: Ribosomal ribonucleic acid; PICRUSt: Phylogenetic Investigation of Communities by Reconstruction of Unobserved States; OUT: Operational taxonomic unit; DNA: Deoxyribonucleic acid; KEGG: Kyoto Encyclopedia of Genes and Genomes; PCR: Polymerase chain reaction; LEfSe: Linear discriminant analysis effect size.
\end{abstract}

\section{Supplementary Information}

The online version contains supplementary material available at https://doi. org/10.1186/s13213-022-01669-5.

Additional file 1.

\section{Acknowledgements}

We thank Jipeng Tian, Xue Gou, Guangmei Yang, Jiao Zhang, Shuhan Yang and Tuo Shen for sample collection. Thanks to Xue Liang, Xin Liang, Biyu He, and Zexu Long for their care and help.

\section{Authors' contributions}

Conceptualization: Haijun Su, Heqin Cao, and Xiongwei Yang. Data collection and sample processing: Xiongwei Yang, Qunyi Guo, Yeying Wang, and Caichun Peng. Study design: Xiongwei Yang, Heqin Cao, and Haijun Su. Investigation,
Xiongwei Yang, Caichun Peng, and Yeying Wang. Statistical analyses: Heqin Cao, Haijun Su, and Xiongwei Yang. Writing —original draft: Heqin Cao. Writing —review and editing: Heqin Cao and Haijun Su. Visualization: Heqin Cao and Xiongwei Yang. Supervision: Haijun Su. Project administration: Haijun Su. Funding acquisition: Haijun Su. All authors have read and agreed to the published version of the manuscript. All authors read and approved the final manuscript

\section{Funding}

This research is funded by National Natural Foundation of China (32060307); Provincial Special Project of Wild Boar for Prevention and Control of ASF by State Forestry and Grassland Administration (2019); Forestry Research Project of Guizhou Province (Linkehe J Zi 2020)07).

\section{Availability of data and materials}

Fifteen healthy wild boars were collected in the forest environment of a karst region in Southwest China, Guizhou Province, China.

\section{Declarations}

Ethics approval and consent to participate

The hunting and autopsy samples were approved by Guizhou Forestry Bureau and Animal Experiment Ethics Committee of Guizhou University.

\section{Consent for publication}

Not applicable.

\section{Competing interests}

The authors declare that they have no competing interests.

\section{Author details}

${ }^{1}$ Forestry College of Guizhou University, Guiyang 550025, China. ${ }^{2}$ Research Center for Bio-diversity and Nature Conservation, Guizhou University, Guiyang 550025, China. ${ }^{3}$ College of Life Science, Guizhou Normal University, Guiyang 550001, China. ${ }^{4}$ Administration of Wildlife and Forest Plants of Guizhou Province, Guiyang 550001, China.

Received: 19 December 2021 Accepted: 15 February 2022 Published online: 06 March 2022

\section{References}

Abdel-Moein KA, Saeed H, Samir A (2015) Novel detection of Helicobacter pylori in fish: A possible public health concern. Acta Tropica 152141-144. https://doi.org/10.1016/j.actatropica.2015.09.005

Becker AAMJ, Hesta M, Hollants J, Janssens GPJ, Huys G (2014). Phylogenetic analysis of faecal microbiota from captive cheetahs reveals underrepresentation of Bacteroidetes and Bifidobacteriaceae. Bmc Microbiol 14. https://doi.org/10.1186/1471-2180-14-43.

Chi C, Liu J-Y, Fei S-Z, Zhang C, Chang Y-Q, Liu X-L, et al. (2014). Effect of intestinal autochthonous probiotics isolated from the gut of sea cucumber (Apostichopus japonicus) on immune response and growth of $\mathrm{A}$. japonicus. Fish Shellfish Immunol 38(2):367-373. https://doi.org/10.1016/j. fsi.2014.04.001.

Crespo-Piazuelo D, Estelle J, Revilla M, Criado-Mesas L, Ramayo-Caldas Y, Ovilo C, et al. (2018). Characterization of bacterial microbiota compositions along the intestinal tract in pigs and their interactions and functions. Scientific Reports 8. https://doi.org/10.1038/s41598-018-30932-6.

David LA, Maurice CF, Carmody RN, Gootenberg DB, Button JE, Wolfe BE et al (2014) Diet rapidly and reproducibly alters the human gut microbiome. Nature 505(7484):559. https://doi.org/10.1038/nature12820

Di Gioia D, Aloisio I, Mazzola G, Biavati B (2014) Bifidobacteria: their impact on gut microbiota composition and their applications as probiotics in infants. Applied Microbiol Biotechnol 98(2):563-577. https://doi.org/10. 1007/s00253-013-5405-9

Fang W, Fang Z, Zhou P, Chang F, Hong Y, Zhang X, et al. (2012). Evidence for lignin oxidation by the giant panda fecal microbiome. Plos One 7(11). https://doi.org/10.1371/journal.pone.0050312. 
Fernando SC, Purvis HT II, Najar FZ, Sukharnikov LO, Krehbiel CR, Nagaraja TG et al (2010) Rumen microbial population dynamics during adaptation to a high-grain diet. Appl Environ Microbiol 76(22):7482-7490. https://doi. org/10.1128/aem.00388-10

Genov PV (2006) A review of the cranial characteristics of the Wild Boar (Susscrofa Linnaeus 1758), with systematic conclusions. Mammal Review 29(4):205-234. https://doi.org/10.1046/j.1365-2907.1999.2940205.x

Guan Y, Yang H, Han S, Feng L, Wang T, Ge J (2017). Comparison of the gut microbiota composition between wild and captive sika deer (Cervus nippon hortulorum) from feces by high-throughput sequencing. Amb Express 7. https://doi.org/10.1186/s13568-017-0517-8.

Guo W, Mishra S, Wang C, Zhang H, Ning R, Kong F, et al. (2019). Comparative Study of Gut Microbiota in Wild and Captive Giant Pandas (Ailuropoda melanoleuca). Genes 10(10). https://doi.org/10.3390/genes10100827.

He F, Zhai J, Zhang L, Liu D, Ma Y, Rong K, et al.(2018). Variations in gut microbiota and fecal metabolic phenotype associated with fenbendazole and ivermectin tablets by $16 \mathrm{~S}$ rRNA gene sequencing and LC/MSbased metabolomics in Amur tiger. Biochem Biophysical Res Commun 499(3):447-453. https://doi.org/10.1016/j.bbrc.2018.03.158.

Henning PH, Horn CH, Steyn DG, Meissner HH, Hagg FM (2010) The potential of Megasphaera elsdenii isolates to control ruminal acidosis. Animal Feed Sci Technol 157(1-2):13-19. https://doi.org/10.1016/j.anifeedsci. 2009.12.011

Hua Y, Cao H, Wang J, He F, Jiang G (2020). Gut microbiota and fecal metabolites in captive and wild North China leopard (Panthera pardus japonensis) by comparsion using $16 \mathrm{~s}$ rRNA gene sequencing and LC/ MS-based metabolomics. Bmc Veterinary Res 16(1). https://doi.org/10. 1186/s12917-020-02583-1.

Huang J, Zhang W, Fan R, Liu Z, Huang T, Li J, et al. (2020). Composition and functional diversity of fecal bacterial community of wild boar, commercial pig and domestic native pig as revealed by $16 \mathrm{~S}$ rRNA gene sequencing. Arch Microbiol 202(4):843-857. https://doi.org/10.1007/ s00203-019-01787-W.

Huang W, Cheng Z, Lei S, Liu L, Lv X, Chen L, et al. (2018). Community composition, diversity, and metabolism of intestinal microbiota in cultivated European eel (Anguilla anguilla). Appl Microbiol Biotechnol 102(9):41434157. https://doi.org/10.1007/s00253-018-8885-9.

Isaacson R, Kim HB (2012). The intestinal microbiome of the pig. Animal Health Res Reviews 13(1):100-109. https://doi.org/10.1017/s1466252312000084.

Ishaq SL, Wright AD(2014). High-Throughput DNA Sequencing of the Ruminal Bacteria from Moose (Alces alces) in Vermont, Alaska, and Norway. Microbial Ecol 68(2):185-195. https://doi.org/10.1007/s00248-014-0399-0.

Jin L, Wang L, Wang Z, Xue B, Peng Q (2019). Analysis of cecum bacterial diversity of goat based on Illumina MiSeq sequencing. Microbiology China 46(006):1423-1433.

Lau SKP, Teng JLL, Chiu TH, Chan E, Tsang AKL, Panagiotou G, et al. (2018). Differential microbial communities of omnivorous and herbivorous cattle in Southern China. Comput Structural Biotechnol J 1654-60. https://doi.org/ 10.1016/j.csbj.2018.02.004.

Li Q, Hu J, Nie Q, Chang X, Fang Q, Xie J et al (2021) Hypoglycemic mechanism of polysaccharide fromCyclocarya paliurusleaves in type 2 diabetic rats by gut microbiota and host metabolism alteration. Sci China-Life Sci 64(1):117-132. https://doi.org/10.1007/s11427-019-1647-6

Li X-Q, Zhu Y-H, Zhang H-F, Yue Y, Cai Z-X, Lu Q-P, et al. (2012). Risks associated with high-dose Lactobacillus rhamnosus in an Escherichia coli model of piglet diarrhoea: intestinal microbiota and immune imbalances. Plos One7(7). https://doi.org/10.1371/journal.pone.0040666.

Liu H, Li L, Ma Q, Wan D, Wan S, Zhu Y (2011) Review on wild boar research. Sichuan J Zool 30(2):5

Looft T, Allen HK, Cantarel BL, Levine UY, Bayles DO, Alt DP et al (2014) Bacteria, phages and pigs: the effects of in-feed antibiotics on the microbiome at different gut locations. Isme J 8(8):1566-1576. https://doi.org/10.1038/ ismej.2014.12

Ma C, Zhang H, Liu C, Zhao J (2014) Research Advance in Ruminal and Intestinal Microbes Diversity in Cattle. Chin J Animal Nutri 26(4):852-862

Mariat D, Firmesse O, Levenez F, Guimaraes VD, Sokol H, Dore J, et al. (2009). The Firmicutes/Bacteroidetes ratio of the human microbiota changes with age. Bmc Microbiol 9. https://doi.org/10.1186/1471-2180-9-123.

McCoy AN, Araujo-Perez F, Azcarate-Peril A, Yeh JJ, Sandler RS, Keku TO (2013). Fusobacterium Is Associated with Colorectal Adenomas. Plos One 8(1). https://doi.org/10.1371/journal.pone.0053653.
Niu Q, Li P, Hao S, Zhang Y, Kim SW, Li H, et al. (2015). Dynamic distribution of the gut microbiota and the relationship with apparent crude fiber digestibility and growth stages in pigs. Scientific Reports 5. https://doi.org/10. 1038/srep09938.

Petrova MI, Lievens E, Malik S, Imholz N, Lebeer S (2015). Lactobacillus species as biomarkers and agents that can promote various aspects of vaginal health. Front Physiol 6. https://doi.org/10.3389/fphys.2015.00081.

Ruggiero P (2014) Use of probiotics in the fight against Helicobacter pylori. World J Gastrointestinal Pathophysiol 5(4):384-391. https://doi.org/10. 4291/wjgp.v5.14.384

Salyers AA, Vercellotti JR, West SE, Wilkins TD (1977). Fermentation of mucin and plant polysaccharides by strains of Bacteroides from the human colon. Appl Environ Microbiol 33(2):319-322. https://doi.org/10.1128/ aem.33.2.319-322.1977.

Shin N-R, Whon TW, Bae J-W (2015) Proteobacteria: microbial signature of dysbiosis in gut microbiota. Trends Biotechnol 33(9):496-503. https://doi. org/10.1016/j.tibtech.2015.06.011

Su H, Hu C, Zhang M, Liang S (2018) A study on the characteristics of crop damage caused by wild boar ( Sus scrofa) and the attitudes of local residents in Chishui Alsophila National Nature Reserve, Guizhou Province. China. Acta Theriologica Sinica 38(4):359-368

Suchodolski JS, Foster ML, Sohail MU, Leutenegger C, Queen EV, Steiner JM, et al. (2015). The Fecal Microbiome in Cats with Diarrhea. Plos One 10(5). https://doi.org/10.1371/journal.pone.0127378.

Suen G, Stevenson DM, Bruce DC, Chertkov O, Copeland A, Cheng J-F et al (2011) Complete genome of the cellulolytic ruminal bacterium Ruminococcus albus 7. J Bacteriol 193(19):5574-5575. https://doi.org/10.1128/ jb.05621-11

Tao S, Bai Y, Zhou X, Zhao J, Yang H, Zhang S et al (2019) In vitro fermentation characteristics for different ratios of soluble to insoluble dietary fiber by fresh fecal microbiota from growing pigs. Acs Omega 4(12):15158-15167. https://doi.org/10.1021/acsomega.9b01849

Thoetkiattikul H, Wuttichai M, Tangphatsornruang S, Pattarajinda V, Eurwilaichitr L, Champreda V (2013) Comparative analysis of microbial profiles in cow rumen fed with different dietary fiber by tagged 165 rRNA gene pyrosequencing. Curr Microbiol 67(2):130-137. https://doi.org/10.1007/ s00284-013-0336-3

Wang J, Wang Q, Zeng D, Niu L, Jian P, Zhang Y et al (2015) Comparison of fecal microbiota from healthy and diaahea Rhinopithecus roxellana. Chinese J Vet Sci 35(08):1232-1238

Wang X, Zhang Y, Wen Q, Wang Y, Wang Z, Tan Z, et al. (2020). Sex Differences in Intestinal Microbial Composition and Function of Hainan Special Wild Boar. Animals 10(9). https://doi.org/10.3390/ani10091553.

Weldon L, Abolins S, Lenzi L, Bourne C, Riley EM, Viney M (2015). The gut microbiota of wild mice. Plos One 10(8). https://doi.org/10.1371/journal. pone. 0134643 .

Wu C, Yang F, Gao R, Huang Z, Xu B, Dong Y et al (2010) Study of fecal bacterial diversity in Yunnan snub-nosed monkey (Rhinopithecus bieti) using phylogenetic analysis of cloned 16S rRNA gene sequences. Afr J Biotechnol 9(38):6278-6289. https://doi.org/10.1186/1475-2859-9-72

Wu X, Zhang H, Chen J, Shang S, Wei Q, Yan J et al (2016) Comparison of the fecal microbiota of dholes high-throughput Illumina sequencing of the V3-V4 region of the $16 \mathrm{~S}$ rRNA gene. Appl Microbiol Biotechnol 100(8):3577-3586. https://doi.org/10.1007/s00253-015-7257-y

Xiong J, Wang K, Wu J, Qiuqian L, Yang K, Qian Y et al (2015) Changes in intestinal bacterial communities are closely associated with shrimp disease severity. Appl Microbiol Biotechnol 99(16):6911-6919. https://doi.org/10. 1007/s00253-015-6632-z

Xue Z, Zhang W, Wang L, Hou R, Zhang M, Fei L, et al. (2015). The bamboo-eating giant panda harbors a carnivore-like gut microbiota, with excessive seasonal variations. Mbio 6 (3). https://doi.org/10.1128/mBio.00022-15.

Yang G, Shi C, Zhang S, Liu Y, Li Z, Gao F et al (2020) Characterization of the bacterial microbiota composition and evolution at different intestinal tract in wild pigs (Sus scrofa ussuricus). Peerj 8. https://doi.org/10.7717/ peerj.9124

Yang H, Huang X, Fang S, Xin W, Huang L, Chen C (2016). Uncovering the composition of microbial community structure and metagenomics among three gut locations in pigs with distinct fatness. Scientific Reports 6. https://doi.org/10.1038/srep27427.

Ye L, Amberg J, Chapman D, Gaikowski M, Liu W-T (2014) Fish gut microbiota analysis differentiates physiology and behavior of invasive Asian carp and 
indigenous American fish. Isme J 8(3):541-551. https://doi.org/10.1038/ ismej.2013.181

Yuan Y, Liu Q, Pei E (2019) Illumina-based analysis of the fecal microbe diversity of sun bears (Helarctos malayanus). Chin J Wildlife 40(4):882-889

Zeng Y, Zeng D, Zhou Y, Niu L, Deng J, Li Y et al (2018) Microbial biogeography along the gastrointestinal tract of a red panda. Front Microbiol 9. https:// doi.org/10.3389/fmicb.2018.01411

Zhang X, Wu Y, Ye H, Feng C, Han D, Tao S et al (2020) Dietary milk fat globule membrane supplementation during late gestation increased the growth of neonatal piglets by improving their plasma parameters, intestinal barriers, and fecal microbiota. Rsc Advances 10(29):16987-16998. https://doi. org/10.1039/d0ra02618b

Zhu J, Dai W, Qiu Q, Dong C, Zhang J, Xiong J (2016) Contrasting ecological processes and functional compositions between intestinal bacterial community in healthy and diseased shrimp. Microbial Ecology 72(4):975-985. https://doi.org/10.1007/s00248-016-0831-8

Zhu L, Wu Q, Dai J, Zhang S, Wei F (2011) Evidence of cellulose metabolism by the giant panda gut microbiome. Proc National Acad Sci U S A 108(43):17714-17719. https://doi.org/10.1073/pnas.1017956108

\section{Publisher's Note}

Springer Nature remains neutral with regard to jurisdictional claims in published maps and institutional affiliations.

- fast, convenient online submission

- thorough peer review by experienced researchers in your field

- rapid publication on acceptance

- support for research data, including large and complex data types

- gold Open Access which fosters wider collaboration and increased citations

- maximum visibility for your research: over 100M website views per year

At BMC, research is always in progress.

Learn more biomedcentral.com/submissions 